\title{
Distribution and molecular analysis of Blastocystis subtypes from gastrointestinal symptomatic and asymptomatic patients in Iran
}

\author{
Asma Delshad ${ }^{1}$, Mehrzad Saraei ${ }^{2,3}$, Safar Ali Alizadeh ${ }^{4,5}$, Soheila Rahimi Niaraki ${ }^{1}$, \\ Mahmood Alipour ${ }^{6}$, Bahram Hosseinbigi ${ }^{4}$, Arezoo Bozorgomid ${ }^{7}$, Elham Hajialilo ${ }^{2,3}$
}

1. Student Research Committee, Qazvin University of Medical Sciences, Qazvin, Iran.

2. Cellular and Molecular Research Center, Research Institute for Prevention of Non- Communicable Disease, Qazvin University of Medical Sciences, Qazvin, Iran.

3. Department of Parasitology and Mycology, Qazvin University of Medical Sciences, Qazvin, Iran.

4. Medical Microbiology Research Center, Qazvin University of Medical Sciences, Qazvin, Iran.

5. Department of Microbiology, Qazvin University of Medical Sciences, Qazvin, Iran.

6. Department of Social Medicine, Qazvin University of Medical Sciences, Qazvin, Iran.

7. Infectious Diseases Research Center, Health Institute, Kermanshah University of Medical Sciences, Kermanshah, Iran

\begin{abstract}
Introduction: Blastocystis is a common intestinal parasite of human and animal hosts. The parasite has 17 subtypes, and among those at least nine subtypes (ST1-ST9) are found in human hosts.

Objective: The aim of the present study was to investigate the presence of different subtypes of Blastocystis spp. among the patients referred to Velayat hospital of Qazvin province, Iran.

Methods: Overall, 864 stool samples were examined by using formalin-ethyl acetate concentration method and Trichrome staining. All specimens were cultured in clotted fetal bovine medium. Later, DNA extraction and PCR amplification of $18 \mathrm{~S}$ ribosomal RNA gene region was conducted and phylogenetic tree constructed.

Results: The results revealed $7.9 \%$ (68/864) of the study population were infected with Blastocystis. Intestinal symptoms were observed in 61\% (36/59) of individuals positive for Blastocystis, with abdominal pain in 58\% (21/36) of cases which was more frequent than other intestinal signs. No significant relationship was observed among the study variables. By molecular and phylogenetic analysis, three subtypes ST1 (45\%), ST2 (30\%) and ST3 (23\%) of parasite were identified.

Conclusion: This study showed ST1 subtype was the predominant subtype among the positive specimens, meanwhile the highest haplotype and nucleotide diversity were clarified in ST3 subtype.

Keywords: Blastocystis, subtype, Phylogenetic analysis, Iran.

DOI: https://dx.doi.org/10.4314/ahs.v20i3.21

Cite as: Delshad A, Saraei M, Alizadeh SA, Niaraki SR, Alipour M, Hosseinbigi B, et al. Distribution and molecular analysis of Blastocystis subtypes from gastrointestinal symptomatic and asymptomatic patients in Iran. Afri Health Sci.2020;20(3): 1179. 1189. bttps:// dx.doi.org/10.4314/abs.v20i3.21
\end{abstract}

\section{Corresponding author:}

Mehrzad Saraei,

Department of Parasitology and Mycology,

Qazvin University of Medical Sciences, Qazvin, Iran

E-mail address: msaraei@qums.ac.ir

and

Elham Hajialilo,

Department of Parasitology and Mycology,

Qazvin University of Medical Sciences, Qazvin, Iran

E-mail address: e.hajialilo@qums.ac.ir

\section{Introduction}

Blastocystis is an anaerobic parasite of human and animal intestinal tract. According to phylogenetic approach, the parasite belongs to Stramenopiles of the Eukaryo$\mathrm{ta}^{1}$. The parasite is a common intestinal microorganism reported in epidemiological surveys ${ }^{2}$. High frequency of this parasite may be due to the reluctance of physicians to treat self-limiting infections or resistance of the parasite to anti-parasitic drugs in conventional treatment, therefore, Blastocystis could easily colonize intestinal empty niches. Moreover, Blastocystis could reside as a non-pathogenic microorganism in the healthy human

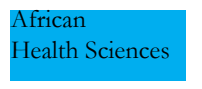

(C) 2020 Delshad A et al. Licensee African Health Sciences. This is an Open Access article distributed under the terms of the Creative commons Attribution License (https://creativecommons.org/licenses/BY/4.0), which permits unrestricted use, distribution, and reproduction in any medium, provided the original work is properly cited. 
$\mathrm{gut}^{3,4}$. The main route of transmission is fecal-oral, but the exact source of transmission is unknown ${ }^{5,6}$. The thick-walled cystic form of the polymorphic parasite is probably a dominant form in external transmission hence, poor sanitation has a major role in infection. Moreover, contamination of food and water sources as well as exposure to animals are considered as other significant risk factors. ${ }^{7,8}$ Non-specific gastrointestinal sings including acute or chronic diarrhea, abdominal pain, vomiting, nausea and irritable bowel syndrome (IBS) may be linked to the infection with this parasite ${ }^{7}$, 9 . Some studies did not reveal a correlation between the occurrence of Blastocystis and IBS, although these studies demonstrated that Blastocystis is less prevalent among IBS patients ${ }^{10,11}$.

Although the parasite is found in symptomatic and asymptomatic individuals, immunocompromised people including HIV/AIDS and cancer patients are clarified as susceptible populations with diarrheal illness. ${ }^{12,13}$ Genetic heterogeneity of Blastocystis based on polymorphic small sub-unit ribosomal DNA (SSU rDNA) gene region has shown the presence of at least seventeen subtypes (STs) in mammals and birds hosts. ${ }^{14}$ The ST1 to ST9 and ST12 are the predominant subtypes in human populations with ST3 as the most common among these subtypes ${ }^{7,15-17}$. Several epidemiological surveys in different parts of Iran have demonstrated the presence of ST1, ST2, ST3, ST4, ST5, and ST7 in human host ${ }^{18,19}$. A study performed in southwest Iran showed ST3 as the predominant subtype with gastrointestinal symptoms. ${ }^{20}$ Another research from Iran reported that the ST2 subtype is highly prevalent among diarrheic patients ${ }^{21}$. The ST1 subtype in gastrointestinal patients, compared to asymptomatic cases, was significantly dominant in $\operatorname{Iran}^{22}$. Available information on different subtypes indicates that each subtype could have a diverse pathogenicity, so investigation and determination of subtypes can help improve knowledge of parasite pathogenicity ${ }^{23-25}$. This study aimed to fill the geographic gap in the information of Blastocystis subtypes and identify the different STs of parasite among the human population of Qazvin, northern margin of central Iran.

\section{Materials and methods \\ Sample collection}

A total of 864 stool samples were collected from the patients referred to Velayat hospital laboratory in Qazvin, for routine stool examination in 2016. A questionnaire was completed with demographic information such as age, sex, and gastrointestinal symptoms of the participants. Ethical approval of the study was obtained from the Medical Ethics Committee of Qazvin University of Medical Sciences (IR. QUMS. REC. 1395. 118).

\section{Sample processing and Blastocystis cultivation}

All specimens were processed and examined in the parasitology laboratory at Qazvin Medical School. Diagnosis of Blastocystis was performed by direct wet-mount and formalin-ethyl acetate concentration method as well as trichrome staining as a confirmatory method for the detection of parasite. Clotted fetal bovine medium was used to culture all specimens. ${ }^{21}$ Briefly, $1 \mathrm{ml}$ of Locke's solution was added to slant clotted fetal bovine serum followed by addition of fresh stool sample $(200 \mathrm{mg}$ ) plus Streptomycin and Nystatin into the culture tubes and finally incubated at $37^{\circ} \mathrm{C}$, under anaerobic condition for ten days. The cultures were followed up every 48 hours up to 10 days by light microscopy and examining the direct smears for growth of Blastocystis. The negative culture was confirmed based on indiscernible growth of parasite after ten days ${ }^{19}$.

\section{DNA extraction and PCR amplification}

DNA extraction was carried out on $200 \mu$ l of liquid phase of positive medium. The samples were centrifuged, pellets harvested for extraction, and then the genomic DNA was extracted using a commercial kit (Stool DNA Isolation mini kit; Yekta Tajhiz Azma, Iran). Small subunit rRNA gene region, that is 18 srRNA was amplified by specific primers of Blastocystis, NC1 (5'-AGCGTATATTAACGTTGTTGCAG -3') and NC2 (5'- TCAATCCTTCCTATGTCTGG -3'). The primers used for Blastocystis were designed by Beacon Designer7 and Primer-BLAST (http://blast.ncbi.nlm. nih.gov/Blast.cgi). A $20 \mu \mathrm{l}$ reaction volume containing $10 \mu \mathrm{l}$ master mix (Amplicon; Taq DNA Polymerase Master Mix RED, Denmark) and template DNA, $0.1 \mu \mathrm{M}$ of each primer and distilled water was used for PCR under following temperature conditions: $95^{\circ} \mathrm{C}$ for $5 \mathrm{~min} ; 35$ cycles of $95^{\circ} \mathrm{C}$ for 20 seconds, annealing step at $64{ }^{\circ} \mathrm{C}$ for 20 seconds, and $72{ }^{\circ} \mathrm{C}$ for 30 seconds; final extension at $72{ }^{\circ} \mathrm{C}$ for 5 minutes. subsequently, the PCR product were stained and electrophoresed on a $1.5 \%$ agarose gel in TBE buffer (Tris, boric acid and EDTA). The specific band was viewed under UV light.

\section{Sequencing analysis}

All PCR products were purified (AccuPrep ${ }^{\circledR}$ PCR/Gel Purification Kit- Bioneer, Korea) and sequenced with ABI 3130X sequencer. The sequences were adjusted manually by Chromas (version 1.0.0.1), and then compared with available reference sequences in GenBank database using NCBI BLAST (Basic Local Alignment 
Search Tool) by software (https://blast.ncbi.nlm.nih. gov/Blast.cgi). All Blastocystis sequences were submitted to the NCBI database under the Accession Numbers MH177914- MH177915, MH220975-MH221018.

\section{Phylogenetic analysis}

ClustalW incorporated in the BioEdit software was used to sequence alignment ${ }^{26}$. Phylogenetic tree was constructed by the MEGA7 software (Molecular and Evolution Genetic Analysis v7), Maximum-Likelihood algorithm with Tamura-3 parameter substitution model was applied. Finally, a bootstrap of 1000 replicates was considered.

\section{Genetic differentiation and haplotype network analysis}

Genetic variability for ribosomal RNA marker was evaluated by the number of haplotypes ( $\mathrm{Hn})$, haplotype diversity $(\mathrm{Hd})$, nucleotide diversity $(\pi)$, number of variable sites (S), number of nucleotide differences $(K)$, neutrality indices including Tajima's D and Fu's Fs statistic using DnaSP v.5.0 ${ }^{27}$. Pairwise fixation index (FST) values obtained from Blastocystis spp populations were calculated using Arlequin 3.5. ${ }^{28} \mathrm{~A}$ median joining network haplotype network ${ }^{29}$ to visualize the relationships among 46 isolates was constructed using PopART software (http://popart.otago.ac.nz).

\section{Population structure}

Genetic structure was assessed by STRUCTURE 2.3.4 ${ }^{30}$. An admixture model with correlated allele frequencies was chosen.en replicates for each $\mathrm{K}$ (from 1 to 4) were computed, with 100,000 Markov chain Monte Carlo (MCMC) iterations after a burn- in of 100,000 iterations. The optimal $\mathrm{K}$ was determined based on the $\Delta \mathrm{K}$ method implemented in Structure Harvester ${ }^{31}$. The STRUCTURE results were calculated using CLUMPAK 1.1.2 ${ }^{32 .}$

\section{Statistical method}

Chi-square test in SPSS software version 17.00 (SPSS Inc., Chicago, IL, USA) was used to determine significance between variables. A p value of $<0.05$ was considered statistically significant for the differences observed.

\section{Results}

Blastocystis was detected in 7.9\% (68/864) and 6.8\% (59/864) of participants using formalin-ethyl acetate concentration and culture methods, respectively. Blastocystis infection was found among both female 7.7\% (38/492) and male 5.6\% (21/372) individuals. The parasite prevalence rate in $>70$-year old age group was higher $8.8 \%(8 / 91)$ than other age groups while no infection was found among 0-9 years old age group. Gastrointestinal signs among positive specimens for Blastocystis was $61 \%(36 / 59)$ whereas $39 \%(23 / 59)$ of the individuals did not show any intestinal symptoms. Abdominal pain with 58\% (21/36) was the most predominant intestinal symptom. Finally, there was no significant relationship between Blastocystis infection and the study variables including gender, age, and gastrointestinal signs (Table 1). All positive specimens in culture demonstrated a specific band by PCR reaction and forty-six isolates were sequenced successfully. According to molecular and phylogenetic analysis presented in Fig. 1, three subtypes (ST1, ST2, and ST3) of Blastocystis were identified in the present study. The percentage and the number of cases detected for 3 different subtypes were $45 \%(21 / 46), 30 \%(14 / 46)$, and 23\% (11/46) for ST1, ST2, and ST3 subtypes, respectively. ST1 was the most prevalent subtype in the present study. Also, ST1 was the dominant subtype in symptomatic patients, nevertheless no significant relationship between the subtypes and gastrointestial symptoms was found. 
Table 1 Socio-demographic features of people referred to Velayat Hospital Lab in Qazvin, Iran.

\begin{tabular}{|c|c|c|c|}
\hline Variable & $\begin{array}{c}\text { Number examined } \\
(\%)\end{array}$ & $\begin{array}{c}\text { Number } \\
\text { infected (\%) }\end{array}$ & P. Value \\
\hline \multicolumn{4}{|l|}{ Sex } \\
\hline Male & $372(43)$ & $21(5.6)$ & \\
\hline Female & $492(57)$ & $38(7.7)$ & 0.2 \\
\hline \multicolumn{4}{|l|}{ Age } \\
\hline $0-9$ & $15(1.76)$ & 0 & \\
\hline $10-19$ & $59(6.92)$ & $2(3.4)$ & \\
\hline $20-29$ & $144(16.82)$ & $10(6.9)$ & \\
\hline $30-39$ & $147(17.2)$ & $8(5.4)$ & \\
\hline $40-49$ & $143(16.7)$ & $10(6.9)$ & \\
\hline
\end{tabular}

0.1

\begin{tabular}{|c|c|c|}
\hline $50-59$ & 161(18.8) & $12(7.5)$ \\
\hline $60-69$ & $96(11.2)$ & $8(8.3)$ \\
\hline $70>$ & $91(10.6)$ & $8(8.8)$ \\
\hline Symptomatic ${ }^{a}$ & $526(64.4)$ & $36(6.8)$ \\
\hline Asymptomatic & 291(35.6) & $22(7.6)$ \\
\hline
\end{tabular}

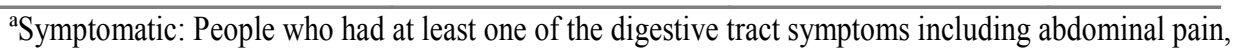
diarrhea, bloody diarrhea, nausea, vomiting. 


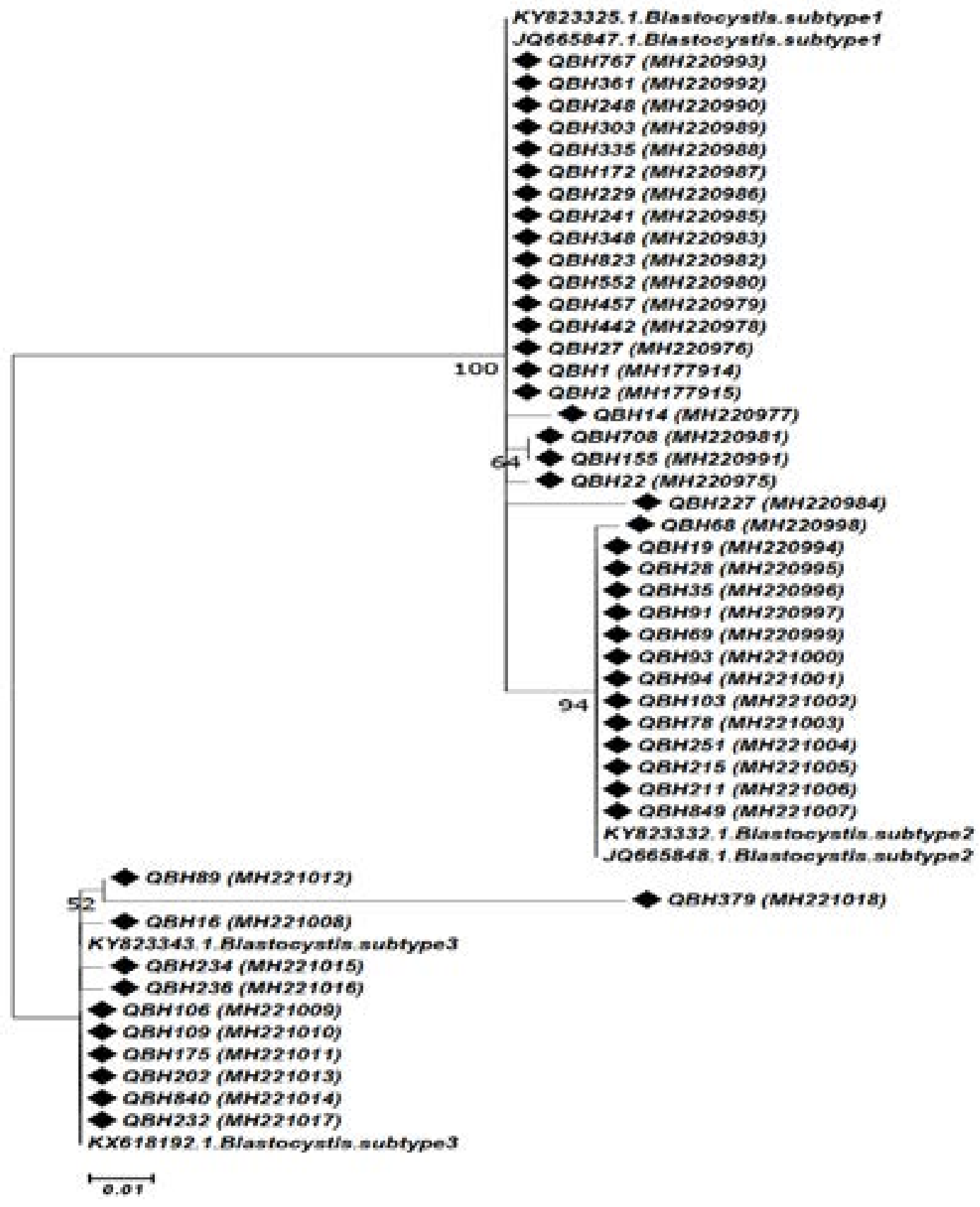

Fig. 1 Phylogenetic tree of Blastocystis spp. isolates from Qazvin, Iran. Three subtypes of Blastocystis were identified in the present study $(\bullet)$, according to phylogenetic analysis of nucleotide sequences. The tree was designed by using the Maximum-Likelihood test and the Tamura 3-parameter model as implemented in the MEGA7 software. 
Genetic diversity indices of Blastocystis spp were shown in Table 2. The number of haplotypes (Hn) was 5, 2 and 6 for ST1, ST2 and ST3, respectively. Highest haplotype and nucleotide diversity $(0.727$ and 0.01728 , respectively) in ST3 were clearly evident in the large values of average number of nucleotide differences. Tajima's D value was negative in all three subtypes, although statistical significance existed for ST1 and ST3. According to Fu's Fs statistic, the only negative value was observed in ST1 which agrees with Tajima's D value, thus it is suggestive of a recent population expansion, although statistically not significant. The range of pairwise Fst estimates among subtypes were high (ranging from 0.75910 to 0.90874 ) and the highest difference was seen between ST2 and ST3 that indicated highest genetic differences between these two subtypes (Table 3). The population structure of the 46 Blastocystis sequence was inferred using STRUCTURE 2.3.4 and the peak of delta $K$ was observed at $K=2$, suggesting the presence of two main populations in the Blastocystis sp. (Fig. 2, 3).

Table 2 Diversity and neutrality indices of Blastocystis spp based on nucleotide sequences of ribosomal RNA gene

\begin{tabular}{ccccccccc}
\hline Subtypes & No & Hn & Hd & $\boldsymbol{\pi}$ & S & K & \multicolumn{2}{c}{ Neutrality indices } \\
\hline & & & & & & & Tajima's D & Fu's Fs \\
\hline ST1 & 21 & 5 & 0.424 & 0.00336 & 9 & 0.94286 & $-2.11446^{*}$ & -1.203 \\
ST2 & 14 & 2 & 0.143 & 0.00052 & 1 & 0.14286 & -1.15524 & 0.937 \\
ST3 & 11 & 6 & 0.727 & 0.01728 & 26 & 4.87273 & $-2.07567^{* *}$ & 0.657 \\
\hline
\end{tabular}

No: number of sequences; Hn: number of haplotypes; $\pi$ : nucleotide diversity; S: Number of variable sites, k: average number of nucleotide differences. ${ }^{*} \mathrm{P}<0.05,{ }^{* *} \mathrm{P}<0.01$

Table 3 Pairwise fixation indices (Fst) of the Blastocystis isolates calculated from nucleotide sequences derived from 18srRNA gene.

\begin{tabular}{|l|l|l|l|}
\hline & ST1 & ST2 & ST3 \\
\hline ST1 & 0.00000 & & \\
\hline ST2 & $0.75910^{*}$ & 0.00000 & \\
\hline ST3 & $0.87641^{*}$ & $0.90874^{*}$ & 0.00000 \\
\hline
\end{tabular}

* Statistically significant $\mathrm{P}<0.05$. 
Fig. 2 Bayesian cluster analysis using the STRUCTURE program: results for $K=2$. Graphical representation of the dataset for the most likely $\mathrm{K}(\mathrm{K}=2)$, where each colour corresponds to a suggested cluster.

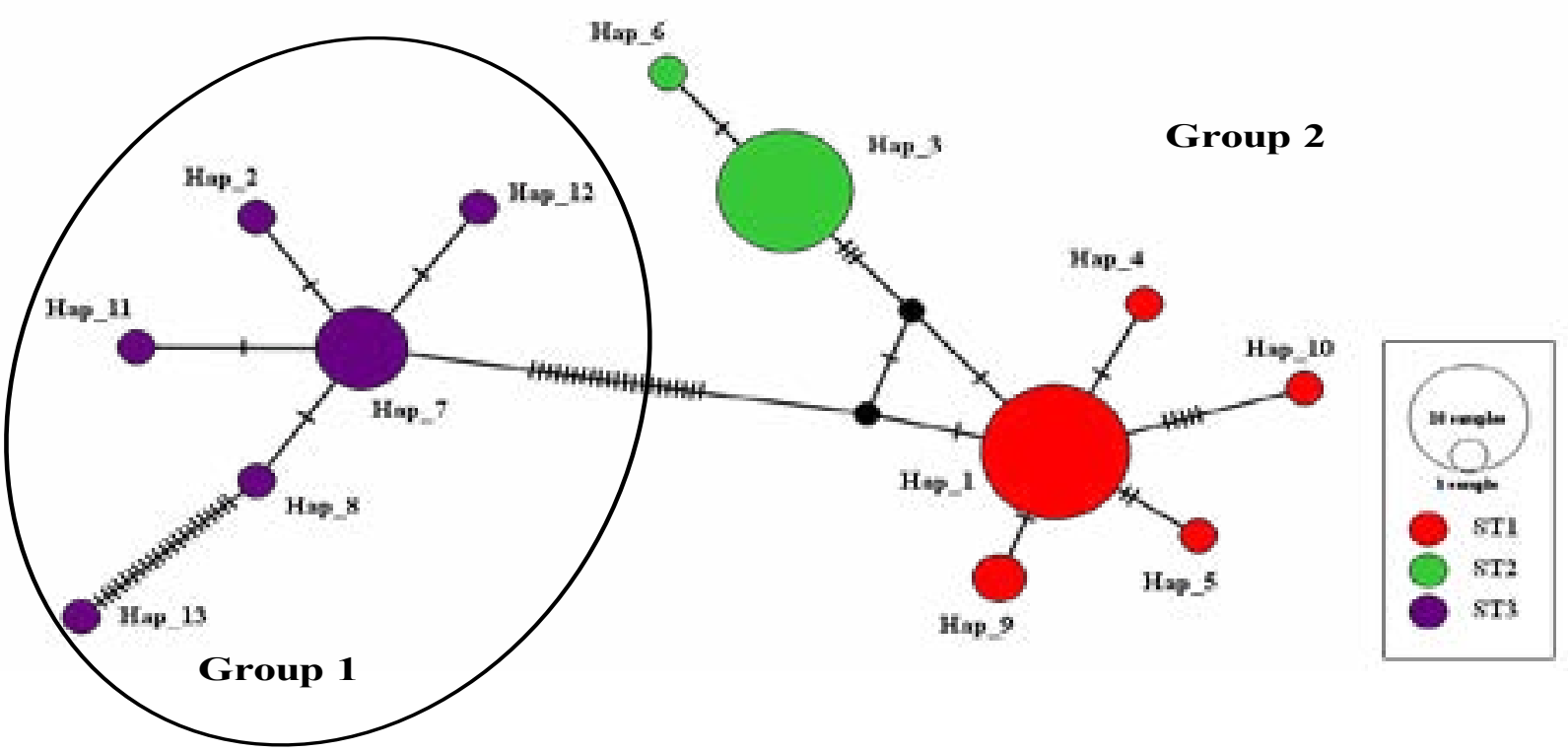

Fig. 3 Haplotype network of Blastocystis population in Qazvin region. Each circle represents a haplotype. Circle sizes are proportional to the corresponding haplotype frequencies. Small black dots represent hypothetical missing and the short lines show the mutational steps. ST1 and ST2 are population (Pop1) and ST3 is Pop2.

\section{Discussion}

Blastocystis is a ubiquitous parasite with worldwide distribution $^{7}$. In the present study this parasite was the most common parasite among stool specimens in which $7.9 \%$ cases of Blastocystis were found by direct microscopic survey although $6.8 \%$ of isolates were positive in the culture method. Variation in results could be due to the polymorphic diversity of the parasite and misdiagnosis with other intestinal parasite and the presence of artifacts. Although the results of our study failed to show a significant relationship between age and Blastocystis, few studies have revealed significant correlation with age variable 33,34 .
In the present study, the ST1, ST2, and ST3 subtypes of the parasite were detected among the Blastocystis positive specimens and these three subtypes were reported to be the most common subtypes in various parts of the country ${ }^{18,19,21,22}$. Moosavi and colleagues showed the presence of ST5 along with three other subtypes in symptomatic and asymptomatic individuals ${ }^{22}$. In another study carried out in south Iran, ST1, ST2, ST3, ST4, ST5 subtypes plus mixed subtypes were observed in human stool specimens ${ }^{20}$. Also, a study from Iran reported ST2, ST3 and ST4 plus mixed subtypes including (ST3 and ST5) and (ST2 and ST3) in patients ${ }^{35}$. The heterogeneity diversity of this parasite is demon- 
strated in human and animal hosts ${ }^{7},{ }^{36}$. Since the pattern of Blastocystis subtypes changes by geographic distribution, it could be reflected in reservoirs and transition pattern among the population ${ }^{37}$. In this context, the ST4 subtype is a good example as it is most prevalent in European patients whereas it is rare in $\mathrm{Asia}^{38-40}$. The predominant subtype in our research was ST1 and several studies similar to the present work have found ST1 as the dominant subtype in the human host $\mathrm{t}^{41,42}$. However, further investigations mainly reported from Iran showed that ST3 was the most common subtype in the human population while the subtype is less common among animals ${ }^{20,22,35,43,44}$. ST1 and ST3 subtypes have a potentially zoonotic transmission ${ }^{45,46}$. According to previous data, ST1 and ST2 were described with low host specificity in animals whereas ST3 subtype is of human origin ${ }^{7,47,48}$. The findings of this study indicate the risk of zoonotic transmission of Blastocystis in these areas which probably arose from different backgrounds including agricultural, animal husbandry, and other activities ${ }^{14,46,49}$. The pathogenicity of Blastocystis is still controversial and not fully understood, due to the presence of parasites in both symptomatic and asymptomatic population. The microbiota communities associated with the parasite may complicate proper explanation of Blastocystis disease ${ }^{18,22,50}$. Some evidence shows that immunocompromised patients are more susceptible to the parasite and develop diarrheal illness. 51,52 Certain subtypes may have a role in the pathgenicity of the parasite, so that a patient with severe intestinal signs may be infected with ST8 subtype of Blastocystis whereas ST4 subtype could be frequently observed among acute diarrhea patients and this, to some extent, could explain the reason for this claim ${ }^{24,53}$. The variant subtypes have a significant association with clinical signs, although in our research a correlation between intestinal signs and the present subtypes was not detected. Similar to our findings, a number of studies failed to reveal any significant relationship ${ }^{40,54,55}$. In contrast, another study showed significant dominance of subtype 1 in irritable bowel syndrome-diarrhea (IBS-D) patients ${ }^{42}$. Consistent with the result of the study by Yakoobt al, a significant domination of ST1, 3 in patients with gastrointestinal symptoms was reported by Moosavi, $2012^{22}$. Also, a research in the Middle East performed by non-quantitative polymerase chain reaction (non-qPCR) showed ST1 was significantly predominant among patients ${ }^{56}$. Despite all information, the genetic diversity over intra- and inter-subtypes variation fails to produce sufficient logical explanations for the presence of any correlation between Blastocystis genotypes and the potential pathogenicity of the parasite ${ }^{19}$.

Results of DnaSP showed that haplotype diversity, nucleotide diversity and number of segregating sites in ST3 was higher compared to ST1 and ST2. This result is supported by a previous study on intra-subtype variations of Blastocystis that revealed high diversity amongst ST3 isolates compared with ST1 and ST2. ${ }^{19}$ According to the 46 Blastocystis sequences, cluster analysis showed that the primary peak of $\Delta \mathrm{K}$ was observed at $\mathrm{K}=2$ (Fig. 2), suggesting that the entire Blastocystis population from Qazvin Province could be divided into two subgroups, population (Pop1 and Pop2). Pop1 includes ST1 sequences and ST2 sequences were clustered together. In contrast, ST3 sequences clustered in Pop2 (Fig 3). To further confirm the above results for the population structure, the existence of two major clusters was supported by the Network. As shown in Fig. 3 , all haplotypes were constructed into two major independent groups. The results suggest that both ST1 and ST2 had a dependent ancestor.

Significant negative results of neutrality tests indicate a recent population expansion. Nevertheless, Tajima's $\mathrm{D}$ and Fu's FS test for neutral evolution of the gene ribosomal RNA had different results which can be due to the heterogeneity of the mutation rate during the nucleotide sequence of the gene or because of a small number of samples. These results imply surplus of the rare mutation across the barcoding region suggesting recent population extension or genetic drift amongst ST3 isolates.

\section{Conclusion}

We conclude that the ST1, ST2, and ST3 subtypes of Blastocystis are the most common subtypes in the study areas, similar to several studies previously reported from Iran. In contrast, several investigations have shown that subtype 1 is the predominant subtype among Blastocystis positive specimens, indicating the presence of geographical genetic variations in the parasite across the country. Thus, health education for high risk population coupled with health promotion could reduce the degree of contamination in the region.

\section{Acknowledgments}

The authors are grateful to the laboratory personnel of Velayat hospital in Qazvin province for their valuable cooperation. We also thank Dr. Ali-Asghar Pahlevan for editing the final version of the English manuscript. 


\section{Funding}

This study was part of an MSc thesis in Qazvin University of Medical Sciences, Qazvin, Iran. The project was financially supported by the Deputy for research and technology of Qazvin University of Medical Sciences.

\section{Conflict of interest}

The authors declare no conflict of interest.

\section{Ethical approval}

This study was approved by the Medical Ethics Committee of Qazvin University of Medical Sciences (Approval code: IR. QUMS. REC. 1395. 118).

\section{Informed consent}

All patients provided written informed consent after explanation of the study purpose and procedures.

\section{References}

1. Arisue N, Hashimoto T, Yoshikawa H, Nakamura Y, Nakamura G, Nakamura F, et al. Phylogenetic position of Blastocystis hominis and of stramenopiles inferred from multiple molecular sequence data. Journal of Eukaryotic Microbiology. 2002;49(1):42-53.

2. Li L-H, Zhou X-N, Du Z-W, Wang X-Z, Wang L-B, Jiang J-Y, et al. Molecular epidemiology of human Blastocystis in a village in Yunnan province, China. Parasitology International. 2007;56(4):281-6.

3. Lokmer A, Cian A, Froment A, Gantois N, Viscogliosi E, Chabé M, et al. Use of shotgun metagenomics for the identification of protozoa in the gut microbiota of healthy individuals from worldwide populations with various industrialization levels. PloS one. 2019;14(2):e0211139.

4. Ramirez-Miranda ME, Hernandez-Castellanos R, Lopez-Escamilla E, Moncada D, Rodriguez-Magallan A, Pagaza-Melero C, et al. Parasites in Mexican patients with irritable bowel syndrome: a case-control study. Parasites \& Vectors. 2010;3(1):96.

5. Leelayoova S, Siripattanapipong S, Thathaisong U, Naaglor T, Taamasri P, Piyaraj P, et al. Drinking water: a possible source of Blastocystis spp. subtype 1 infection in schoolchildren of a rural community in central Thailand. The American Journal of Tropical Medicine and Hygiene. 2008;79(3):401-6.

6. Lee LI, Chye TT, Karmacharya BM, Govind SK. Blastocystis sp.: waterborne zoonotic organism, a possibility? Parasites \& Vectors. 2012;5(1):130.

7. Tan KS. New insights on classification, identification, and clinical relevance of Blastocystis spp. Clinical $\mathrm{Mi}$ crobiology Reviews. 2008;21(4):639-65.
8. Belleza MLB, Cadacio JLC, Borja MP, Solon JAA, Padilla MA, Tongol-Rivera PN, et al. Epidemiologic study of Blastocystis infection in an urban community in the Philippines. Journal of Environmental and Public Health. 2015;2015.

9. Poirier P, Wawrzyniak I, Vivarès CP, Delbac F, El Alaoui H. New insights into Blastocystis spp.: a potential link with irritable bowel syndrome. PLoS Pathogens. 2012;8(3):e1002545.

10. Krogsgaard LR, Engsbro AL, Stensvold CR, Nielsen HV, Bytzer P. The prevalence of intestinal parasites is not greater among individuals with irritable bowel syndrome: a population-based case-control study. Clinical Gastroenterology and Hepatology. 2015;13(3):507-13. e2. 11. Tungtrongchitr A, Manatsathit S, Kositchaiwat C, Ongrotchanakun J, Munkong N, Chinabutr P, et al. Blastocystis hominis infection in irritable bowel syndrome patients. Southeast Asian Journal of Tropical Medicine and Public Health. 2004;35:705-10.

12. Tan T, Ong S, Suresh K. Genetic variability of Blastocystis sp. isolates obtained from cancer and HIV/AIDS patients. Parasitology Research. 2009;105(5):1283.

13. Lepczyńska M, Białkowska J, Dzika E, Piskorz-Ogórek K, Korycińska J. Blastocystis: how do specific diets and human gut microbiota affect its development and pathogenicity? European Journal of Clinical Microbiology \& Infectious Diseases. 2017;36(9):1531-40.

14. Cian A, El Safadi D, Osman M, Moriniere R, Gantois N, Benamrouz-Vanneste $\mathrm{S}$, et al. Molecular epidemiology of Blastocystis sp. in various animal groups from two French zoos and evaluation of potential zoonotic risk. PloS one. 2017;12(1):e0169659.

15. Stensvold CR, Clark CG. Current status of Blastocystis: a personal view. Parasitology International. 2016;65(6):76371.

16. Wong KH, Ng G, Lin RT, Yoshikawa H, Taylor MB, Tan KS. Predominance of subtype 3 among Blastocystis isolates from a major hospital in Singapore. Parasitology Research. 2008;102(4):663-70.

17. Ramírez JD, Sánchez A, Hernández C, Flórez C, Bernal MC, Giraldo JC, et al. Geographic distribution of human Blastocystis subtypes in South America. Infection, Genetics and Evolution. 2016;41:32-5 PubMed.

18. Shahbazi A, Fallah E, Heydarian P, Ghazanchaei A, Khanmohammadi M, Mirsamdi N. PCR-Based Subtyping of Blastocystis Isolates from Symptomatic and Asymptomatic Patients in North-West of Iran. Journal of Pure and Applied Microbiology. 2013;7(4):2957-63.

19. Alinaghizade A, Mirjalali H, Mohebali M, Stensvold $\mathrm{CR}$, Rezaeian M. Inter-and intra-subtype variation of 
Blastocystis subtypes isolated from diarrheic and non-diarrheic patients in Iran. Infection, Genetics and Evolution. 2017;50:77-82.

20. Khademvatan S, Masjedizadeh R, Yousefi-Razin E, Mahbodfar H, Rahim F, Yousefi E, et al. PCR-based molecular characterization of Blastocystis hominis subtypes in southwest of Iran. Journal of Infection and Public Health. 2018;11(1):43-7.

21. Jalallou N, Iravani S, Rezaeian M, Alinaghizade A, Mirjalali H. Subtypes Distribution and Frequency of Blastocystis sp. Isolated from Diarrheic and Non-diarrheic Patients. Iranian Journal of Parasitology. 2017;12(1):63. 22. Moosavi A, Haghighi A, Mojarad EN, Zayeri F, Alebouyeh M, Khazan $\mathrm{H}$, et al. Genetic variability of Blastocystis sp. isolated from symptomatic and asymptomatic individuals in Iran. Parasitology Research. 2012;111(6):2311-5.

23. Stensvold CR, Alfellani M, Clark CG. Levels of genetic diversity vary dramatically between Blastocystis subtypes. Infection, Genetics and Evolution. 2012;12(2):263-73. 24. Stensvold CR, Christiansen DB, Olsen KEP, Nielsen HV. Blastocystis sp. subtype 4 is common in Danish Blastocystis-positive patients presenting with acute diarrhea. The American Journal of Tropical Medicine and Hygiene. 2011;84(6):883-5.

25. Scanlan PD. Blastocystis: past pitfalls and future perspectives. Trends in Parasitology. 2012;28(8):327-34.

26. Hall TA, editor BioEdit: a user-friendly biological sequence alignment editor and analysis program for Windows 95/98/NT. Nucleic acids symposium series; 1999: [London]: Information Retrieval Ltd., c1979-c2000.

27. Librado P, Rozas J. DnaSP v5: a software for comprehensive analysis of DNA polymorphism data. BioInformatics. 2009;25(11):1451-2. PubMed

28. Excoffier L, Lischer HE. Arlequin suite ver 3.5: a new series of programs to perform population genetics analyses under Linux and Windows. Molecular Ecology Resources. 2010;10(3):564-7.

29. Bandelt H-J, Forster P, Röhl A. Median-joining networks for inferring intraspecific phylogenies. Molecular Biology and Evolution. 1999;16(1):37-48.

30. Pritchard JK, Stephens M, Donnelly P. Inference of population structure using multilocus genotype data. Genetics. 2000;155(2):945-59.

31. Earl DA. STRUCTURE HARVESTER: a website and program for visualizing STRUCTURE output and implementing the Evanno method. Conservation Genetics Resources. 2012;4(2):359-61.

32. Kopelman NM, Mayzel J, Jakobsson M, Rosenberg NA, Mayrose I. Clumpak: a program for identify- ing clustering modes and packaging population structure inferences across K. Molecular Ecology Resources. 2015;15(5):1179-91.

33. El Safadi D, Cian A, Nourrisson C, Pereira B, Morelle $\mathrm{C}, \mathrm{B}$ astien $\mathrm{P}$, et al. Prevalence, risk factors for infection and subtype distribution of the intestinal parasite Blastocystis sp. from a large-scale multi-center study in France. BMC Infectious Diseases. 2016;16(1):451.

34. Beyhan YE, Yilmaz H, Cengiz ZT, Ekici A. Clinical significance and prevalence of Blastocystis hominis in Van, Turkey. Saudi Medical Journal. 2015;36(9):1118.

35. Badparva E, Sadraee J, Kheirandish F, Frouzandeh M. Genetic diversity of human blastocystis isolates in khorramabad, central iran. Iranian Journal of Parasitology. 2014;9(1):44.

36. Clark CG, van der Giezen M, Alfellani MA, Stensvold CR. Recent developments in Blastocystis research. Advances in parasitology. 82: Elsevier, 2013. p. 1-32.

37. Li LH, Zhang XP, Lv S, Zhang L, Yoshikawa H, Wu Z, et al. Cross-sectional surveys and subtype classification of human Blastocystis isolates from four epidemiological settings in China. Parasitology Research. 2007;102(1):83-90.

38. Alfellani MA, Stensvold CR, Vidal-Lapiedra A, Onuoha ESU, Fagbenro-Beyioku AF, Clark CG. Variable geographic distribution of Blastocystis subtypes and its potential implications. Acta Tropica. 2013;126(1):11-8.

39. Forsell J, Granlund M, Stensvold CR, Clark G, Evengård B. Subtype analysis of Blastocystis isolates in Swedish patients. European Journal of Clinical Microbiology \& Infectious Diseases. 2012;31(7):1689-96.

40. Souppart L, Sanciu G, Cian A, Wawrzyniak I, Delbac F, Capron M, et al. Molecular epidemiology of human Blastocystis isolates in France. Parasitology Research. 2009;105(2):413.

41. Abdulsalam AM, Ithoi I, Al-Mekhlafi HM, Al-Mekhlafi AM, Ahmed A, Surin J. Subtype distribution of Blastocystis isolates in Sebha, Libya. PLoS One. 2013;8(12):e84372.

42. Yakoob J, Jafri W, Beg MA, Abbas Z, Naz S, Islam $\mathrm{M}$, et al. Irritable bowel syndrome: is it associated with genotypes of Blastocystis hominis. Parasitology Research. 2010;106(5):1033-8.

43. Alfellani MA, Jacob AS, Perea NO, Krecek RC, Taner-Mulla D, Verweij JJ, et al. Diversity and distribution of Blastocystis sp. subtypes in non-human primates. Parasitology. 2013;140(8):966-71.

44. Stensvold CR, Alfellani MA, Nørskov-Lauritsen S, Prip K, Victory EL, Maddox C, et al. Subtype distribution of Blastocystis isolates from synanthropic and zoo 
animals and identification of a new subtype. International Journal for Parasitology. 2009;39(4):473-9.

45. Tan TC, Tan PC, Sharma R, Sugnaseelan S, Suresh KG. Genetic diversity of caprine Blastocystis from Peninsular Malaysia. Parasitology Research. 2013;112(1):85-9. 46. Wang J, Gong B, Yang F, Zhang W, Zheng Y, Liu A. Subtype distribution and genetic characterizations of Blastocystis in pigs, cattle, sheep and goats in northeastern China's Heilongjiang Province. Infection, Genetics and Evolution. 2018;57:171-6 PubMed .

47. Noël C, Dufernez F, Gerbod D, Edgcomb VP, Delgado-Viscogliosi P, Ho L-C, et al. Molecular phylogenies of Blastocystis isolates from different hosts: implications for genetic diversity, identification of species, and zoonosis. Journal of Clinical Microbiology. 2005;43(1):348-55. 48. Yoshikawa H, Abe N, Wu Z. PCR-based identification of zoonotic isolates of Blastocystis from mammals and birds. Microbiology. 2004;150(5):1147-51. PubMed 49. Parkar U, Traub RJ, Vitali S, Elliot A, Levecke B, Robertson I, et al. Molecular characterization of Blastocystis isolates from zoo animals and their animal-keepers. Veterinary Parasitology. 2010;169(1-2):8-17.

50. Andersen LOB, Stensvold CR. Blastocystis in health and disease: are we moving from a clinical to a public health perspective? Journal of Clinical Microbiology. 2016;54(3):524-8.

51. Germani Y, Minssart P, Vohito M, Yassibanda S,
Glaziou P, Hocquet D, et al. Etiologies of acute, persistent, and dysenteric diarrheas in adults in Bangui, Central African Republic, in relation to human immunodeficiency virus serostatus. The American Journal of Tropical Medicine and Hygiene. 1998;59(6):1008-14.

52. Prasad K, Nag V, Dhole T, Ayyagari A. Identification of enteric pathogens in HIV-positive patients with diarrhoea in northern India. Journal of Health, Population and Nutrition. 2000:23-6.

53. Stensvold C, Arendrup M, Nielsen H, Bada A, Thorsen S. Symptomatic infection with Blastocystis sp. subtype 8 successfully treated with trimethoprim-sulfamethoxazole. Annals of Tropical Medicine \& Parasitology. 2008;102(3):271-4.

54. Dogruman-Al F, Dagci H, Yoshikawa H, Kurt Ö, Demirel M. A possible link between subtype 2 and asymptomatic infections of Blastocystis hominis. Parasitology Research. 2008;103(3):685-9.

55. Rahimi Niaraki S, Hajialilo E, Delshad A, Alizadeh SA, Alipour M, Heydarian P, Saraei M. Molecular epidemiology of Blastocystis spp. in children referred to Qods hospital in northwest of Iran. Journal of Parasitic Diseases. 2020;44(1):151-158.

56. El Safadi D, Meloni D, Poirier P, Osman M, Cian A,Gaayeb L, et al. Molecular epidemiology of Blastocystis in Lebanon and correlation between subtype 1 and gastrointestinal symptoms. The American Journal of Tropical Medicine and Hygiene. 2013;88(6):1203-6. 